\title{
Disaster Management and Information Systems: Insights to Emerging Challenges
}

\author{
Ghassan Beydoun $^{1} \cdot$ Sergiu Dascalu ${ }^{2} \cdot$ Dale Dominey-Howes $^{3} \cdot$ Andrew Sheehan $^{4}$
}

Published online: 5 July 2018

(C) Springer Science+Business Media, LLC, part of Springer Nature 2018

\section{Introduction}

Effective Disaster Management (DM) relies on a complex set of interrelated activities and these activities are often knowledge intensive, and time sensitive (Othman and Beydoun 2011). The information systems (IS) community continues to make inroads to enhance the use of technologies to support disaster management. The community continues to identify and extend the relevant theories, and to construct new paradigms that can be brought to bear on the adoption and diffusion of information systems for disaster management. An earlier special issue of the journal of Information Systems Frontiers in 2010 made significant contributions in this space, highlighting the need for extending coordination theory (Janssen et al. 2010), exposing the limits of several organizational theories (Bharosa et al. 2010) and setting the scene to extend diffusion theories for DM inter-organizational information systems (Fedorowicz and Gogan 2010). This special issue continues this discourse.

Ghassan Beydoun

ghassan.beydoun@uts.edu.au

Sergiu Dascalu

dascalus@cse.unr.edu

Dale Dominey-Howes

dale.dominey-howes@sydney.edu.au

Andrew Sheehan

asheehan37@hotmail.com

1 School of System, Management and Leadership, University of Technology Sydney, Ultimo, Australia

2 Department of Computer Science and Engineering, University of Nevada, Reno, NV, USA

3 School of Geosciences, The University of Sydney, Camperdown, Australia

4 Flood Partnership | Waterways and Land, Melbourne Water, Melbourne, Australia
Challenges in inter-organizational and cross disciplinary communication remain a motivating factor in IS research and DM. However, this special issue treats the extension of some potent theories with IS readiness assessment constructs. One focus in this special issue is how do we use such theories to provide assessment methods of DM readiness? The special issue also responds to the emerging theoretical challenges associated with integration of social media in DM information systems. Since the special issue of Information Systems Frontiers appeared in 2010, semantic technologies in the context of social media applications, have considerably progressed. This technical and practical angle on DM and IS receives much attention here. Whilst lack of data standardization and semantics continue to hinder interoperability, social media and improved analysis provide a new vantage point to address these challenges. With recent developments in semantic technologies, and further analysis of related organizational theories, interoperability and IS readiness issues in DM are tackled with new and added vigor.

In this short editorial paper, we first review the content of the issue against the recent developments outlined above. We reflect on the research paradigms on the nexus of DM and IS and conclude with further remarks on what an agenda for the next wave of IS efforts in this space could include.

\section{Reviewing the content}

This special issue of Information Systems Frontiers provides a renewed research vigor in IS and DM. On the theoretical front, it tackles the question of assessing organizational readiness in the face of an emergency. With the growing prevalence of social media, it also provides fresh theoretical and practical perspectives of suitable semantic enrichment of interactions between various stakeholders across different IS platforms. Indeed, from the number of submissions received on social media, it is clear that social media platforms are providing an extremely fertile research ground to investigate those issues. Social media not only provides a very large number of data 
sources, but also a higher degree of heterogeneity of platforms. In what follows, the content of this issue will be reviewed and the contribution of each paper foreshadowed and framed within the identified recent IS innovation trends.

\subsection{Assessment of DM readiness}

Innovations in DM practices require acceptance by the stakeholders involved. Assessing user acceptance and engagement with the IT artefact is crucial before any assumptions are made about the future effectiveness of the IS innovation. Whether the innovation is technological or managerial, effectiveness requires a proper assessment of the potential integration with existing organisational processes and this is clearly underpinned by an appropriate acceptance and engagement of users and practitioners within DM. The first two papers (Wang et al. 2018; Weidinger et al. 2018) deal with this assessment issue. Wang et al. (2018) proposes a DM capability model to assess the DM maturity of an organization. It is based on eight criteria: leadership, resource management, communication, risk management, coordination, planning, exercise program and incident management. The maturity model mirrors the CMM software development maturity model. This assessment model provides an innovative approach to assess how ready an organization is in facing emergencies. It is domain agnostic and enables assessing any organization whether it deals with floods, bushfires or any other incident types. A capability assessment model, namely, DM and control model, is proposed to provide a general guideline for different types of emergency management organizations. Eight indicators are proposed for assessing the capability of the organization. The assessment process includes collecting, analysing, reporting and planning. Weidinger et al. (2018) tackle the same important question as Wang et al. (2018), but the theoretical basis is innovation diffusion theory from an IS perspective. The paper presents a qualitative study of German fire departments to gain insights into the perceived potential of selected emerging technologies from that perspective.

\subsection{Social media}

A key technological innovation since the previous special issue in Information Systems Frontiers appeared in 2010, is the emergence of Social Media platforms (SM). Successful use of SM platforms relies on the behaviour of social media users. There is clear utility for these platforms to facilitate coordination and communication. Particularly, their utility is evident during disaster monitoring and emergency response. However, social media participants are complex social entities, contributing in different ways to their collective task and creating varying participation patterns through social media (Ogie et al. 2018). Understanding these patterns is critical to unlock the SM potential in DM. The next three papers (Liu and Xu 2018; Abedin and Babar 2018; Ogie et al. 2018) focus on SM and DM. The papers by Liu and Xu (2018) and by Abedin and Babar (2018) offer new theoretical insights and expose the limits of some existing IS theories. Whereas, the paper by Ogie et al. (2018) analyses the impact of a rolled out IS application using SM in Jakarta to identify some interesting insights on various types of SM patterns that could point the way for developing new IS theories.

Paper 3 by Liu and Xu (2018) provides an innovative theoretical perspective to enable effective analysis of online posts in the context of a disaster. Structuration theory is used to offer insights on reinforcing emerging and useful roles of an online community during an emergency.

Paper 4 by Abedin and Babar (2018) examines the use of microblogging platforms by emergency response organisations. It highlights the potential use of Twitterand identifies a need for different theories to analyse the use of tweets from organisation users versus the usage of tweets from outside DM oganisational boundaries (e.g. by public or volunteers). A recent event, the bushfires in Victoria, Australia, are used as a case point. While traditionally theories such as World System Theory and Institutional Theory focus on the role of powerful institutional information outlets, platforms like Twitter seem to challenge such notions by sharing the power between institutional and noninstitutional players in the dissemination of disaster information.

Paper 5 by Ogie et al. (2018) also considers how to integrate social media from outside DM organizational boundaries. Indeed, their empirical evidence is consistent with the findings of Abedin and Babar (2018). They uncover various categories of external social media participants and identify their corresponding motivation and levels of reliability for disaster management. Their results have significant implications for developing appropriate IS theories for framing expectations and developing reliance on the use of social media for disaster management. The analysis by Ogie et al. (2018) is rooted in empirical data analysis of flood-related information contributed by Twitter users in Jakarta during the 2014/2015 and 2015/2016 monsoonal seasons.

\subsection{Stakeholder analysis}

To integrate technological innovations, it is important to understand the role of human stakeholders in the process. Agent modelling is a powerful tool to enable better understanding as agents are an excellent metaphor to apply to simulate the autonomous and situated stakeholders in a dynamic environment. The last three papers focus on this.

Paper 6 by Sharma et al. (2018) uses a multi-agent system simulation to predict and adjust evacuation procedures. The paper acknowledges the fuzzy and collective nature of disaster response actions. In enhancing typical agent based simulations, with fuzzy actions, the work pushes the boundaries of agent 
based simulations in this context. The fuzzy and uncertain features of human actions in a disaster setting is further highlighted in the work presented by Kamyabniya et al. (2018) in Paper 7. Kamyabniya et al. (2018) focus on the critical actions in the management of medical resources (blood bank resources) in this context. Despite their clear importance in all types of emergencies, few works in the past have looked at these types of actions. Paper 8 by Inan et al. (2018) concludes the special issue with a theme that can be seen as a gateway to apply all the innovations described in this issue. It tackles the problem of how to best analyze the requirements in a way to identify system needs. It promotes the use of agent oriented analysis not only to support applications such as those presented in Sharma et al. (2018) and Kamyabniya et al. (2018), but to also manage DM knowledge sources more broadly. Such knowledge sources often describe stakeholders, their roles and constraints in DM. All these constructs align well with agent based concepts that underpin agent oriented analysis and modelling activities.

\section{Concluding remarks}

What is clear from this special issue is that research endeavors in DM often requires a focus on a particular disaster setting. This focus enables the validation of the conceptual efforts produced with input from DM practitioners. The collection of papers are rooted in floods, bushfires and firefighting. The focus on flood and firefighting is not surprising, given that there are commonly well established institutions in place to manage these emergency types. Hence, access to case studies and data will always be easier in these two. Whilst most papers will continue to focus on firefighting and flood management, there are other types of disasters that are not covered as much and are not reflected in this special issue including for example, earthquakes and landslides.

This special issue provides a forum for IS academics and DM practitioners to identify and share the challenges, opportunities, and solutions that improve disaster management systems. A particular focus is on the view point of increased use of semantics to enhance information exchange services. We seek to provide a forum to disseminate work aiming to enhance disaster management systems from the perspective of combining semantics with services including social media. This special issues aims to enhance the theoretical understanding of such systems and their use and application in DM.

\section{References}

Abedin, B., Babar, A. (2018). Institutional vs. Non-institutional Use of Social Media during Emergency Response: A Case of Twitter in 2014 Australian Bush Fire. Information Systems Frontiers, 20(4). https://doi.org/10.1007/s10796-017-9789-4.
Bharosa, N., Lee, J. K., \& Janssen, M. (2010). Challenges and Obstacles in Sharing and Coordinating Information during Multi-agency Disaster Response: Propositions from Field Exercises. Information Systems Frontiers, 12(1), 49-65.

Fedorowicz, J., \& Gogan, J. L. (2010). Reinvention of Interorganizational Systems: A Case Analysis of the Diffusion of a Bio-terror Surveillance System. Information Systems Frontiers, 12(1), 81-95 Springer.

Inan, D. I., Beydoun, G., Opper S. (2018). Agent-Based Knowledge Analysis Framework in Disaster Management, Information Systems Frontiers, 20(4). https://doi.org/10.1007/s10796-0179792-9.

Janssen, M., Lee, J. K., \& Bharosa, N. (2010). Advances in Multi-agency Disaster Management: Key Elements in Disaster Research. Information Systems Frontiers, 12(1), 1-7 Springer.

Kamyabniya, A., Lotfi, M. M., Naderpour, M., Yih, Y. (2018). Robust Platelet Logistics Planning in Disaster Relief Operations under Uncertainty: A Coordinated Approach. Information Systems Frontiers, 20(4). https://doi.org/10.1007/s10796-017-9788-5.

Liu, F., Xu, D. (2018). Social Roles and Consequences in Using SM in Disasters: A Structurational Perspective. Information Systems Frontiers, 20(4). https://doi.org/10.1007/s10796-017-9787-6.

Ogie, R. I., Forehead, H., Clarke, R. J., Perez, P. (2018). Participation Patterns and Reliability of Human Sensing in Crowd-Sourced Disaster Management. Information Systems Frontiers, 20(4). https://doi.org/10.1007/s10796-017-9790-y.

Othman, S. H., \& Beydoun, G. (2011). A disaster management Metamodel (DMM) evaluated in 11th international workshop on knowledge management and Acquisition for Smart Systems and Services. 2PKAW 2010. LNCS (LNAI), 6232, 111-125 Springer.

Sharma, S., Ogunlana, K., Scribner, D., Grynovicki, J. (2018). Modeling Human Behavior during Emergency Evacuation using Intelligent Agents: A Multi-Agent Simulation Approach. Information Systems Frontiers, 20(4). https://doi.org/10.1007/s10796-017-9791-x.

Wang, X., Sugumaran, V., Zhang H. (2018). A Capability Assessment Model for Emergency Management Organizations. Information Systems Frontiers, 20(4). https://doi.org/10.1007/s10796-017-9786-7.

Weidinger, J., Schlauderer, S., Overhage, S. (2018). Is the Frontier Shifting into the Right Direction? A Qualitative Analysis of Acceptance Factors for Novel Firefighter Information Technologies. Information Systems Frontiers, 20(4). https://doi. org/10.1007/s10796-017-9785-8.

Professor Ghassan Beydoun received a degree in Computer Science and a Ph.D. degree in Knowledge Systems from the University of New South Wales. He is currently a Professor at the Systems, Management and Leadership at the University of Technology Sydney. He has authored more than 150 papers for international journals and conferences. His research projects are sponsored by Australian Research Council, NSW State Government and the private sector. He investigates the best uses of models in developing methodologies for distributed intelligent systems. His other research interests include cloud adoption, disaster management, ontologies and decisions support systems and their applications, and knowledge acquisition.

Sergiu Dascalu is a Professor in the Computer Science and Engineering Department at the University of Nevada, Reno (UNR), United States of America. He received a Master's degree in Automated Control and Computers from the Polytechnic University of Bucharest, Romania in 1982 and a PhD in Computer Science from Dalhousie University, Halifax, Nova Scotia, Canada in 2001. His main research interests are in software engineering and human-computer interaction, in particular in software approaches and tools for scientific research, simulation environments, virtual reality, and interaction design. Sergiu is the Director of the Software 
Engineering Laboratory (SOELA) at UNR and serves as leader of the Nevada cyber-infrastructure team in a 5-year \$24-million NSF EPSCoR project aimed at improving the understanding of the linkages (or nexus) among solar energy production, related water consumption, and their effects on the environment. In addition, he is the Nevada lead for model and data interoperability in another NSF-funded project that involves watershed research for the US Western Consortium of Idaho, Nevada, and New Mexico. He has served as investigator or co-investigator on several other projects funded by US federal agencies such as NASA, NSF, ONR, as well as by industry organizations such as General Electric and Bally Technologies. Sergiu Dascalu has over 150 peer-reviewed publications and has been involved in the organization of many international conferences and workshops. Furthermore, he was an invited speaker at various universities and events in the US and abroad. Since joining UNR in 2002 he has advised over $10 \mathrm{PhD}$ and 40 Master students. He is the recipient of several professional awards and recognitions, including the 2009 Nevada Center of Entrepreneurship and Technology Faculty Award, the 2011 UNR Distinguished Teacher of the Year Award, and the 2014 UNR College of Engineering's Faculty Excellence Award.

Dale Dominey-Howes is an Associate Professor at the Faculty of Science at the University of Sydney. He completed a BSc (Hons) in Geography and Archaeology at London University and completed his $\mathrm{PhD}$ in geohazards (tsunamis, volcanic eruptions and earthquakes) in Greece at Coventry University, UK under the supervision of Professors David Smith and Alistair Dawson. His PhD was funded by an EU grant enabling him to be based at the National Observatory of Athens under the guidance of Professor Gerassimos Papadopoulos. Dale's interests and expertise are in natural hazards, hazard, risk and vulnerability assessment, disaster and emergency management. He is particularly interested in the interconnections between biophysical systems and the socio-economic contexts in which disasters unfold and considers 'natural hazards' in terms of coupled human-environment systems and policy. He has worked on natural hazards such as earthquakes, river floods, tropical cyclones, tsunami, volcanic eruptions, bushfires and epidemics/pandemic risks in places as diverse as Australia, New Zealand, Bangladesh, India, Greece,
Turkey, Ireland, Papua New Guinea, Fiji, Thailand, Iceland and the Maldives. At the present time, he is involved in a variety of research projects focusing on hazards and risk in the Australasian and IndoPacific regions. Dale has completed research projects and consultancies for organisations as diverse as the United Nations, The World Bank, major insurance and reinsurance companies, State and Federal government departments and risk/disaster management agencies. He currently serves as an advisor to disaster management agencies and policy branches at a range of domestic levels in Australia and was Chairman of the United Nations UNESCO-IOC Post-disaster Policy and Protocols Working Group (2010 - 2014). Dale is also an Editorial Board member of Nature's Scientific Reports and The Anthropocene.

Andrew Sheehan is an emergency management specialist with particular expertise in disaster risk reduction, emergency management planning, intelligence, flood intelligence, flood warning, policy and aid work Water, Sanitation and Hygiene (WASH). As an aid worker with Australian Australian Red Cross Andy has led international deployments to Hue, Vietnam (Typhoon Ketsana, October 2009), Ambrym, Vanuatu (WASH project, 2011), Honiara, Solomon Islands (WASH in emergencies training, 2014), Apia, Samoa (Regional Disaster Response Team Training, 2015) and Suva, Fiji (Pacific Readiness Workshop, 2015). Andy has experience working in remote indigenous communities in Australia and also worked in Kiribati in 2007-2008 on a World Bank climate change adaption program and a NZAid Sustainable Towns Program. In the Victorian emergency management sector Andy led the development and delivery of the inaugural Intelligence Officer training package first delivered in 2015 to emergency managers in Victoria. He has led the development of a number of policies within the flood management sphere and implemented a number of business improvement strategies including enhancing organisational capacity and capability in flood emergency planning, flood warning, flood mapping and information systems relating to flood intelligence. Andy has authored a number of publications and has led peer reviews for publications for the Australian Water Association. In 2014 Andy was awarded most outstanding presentation at the Floodplain Management Association Conference. 